\title{
The acquisition of aquatic skills in preschool children: deep versus shallow water swimming lessons
}

\author{
Helena A. Rocha ${ }^{1}$, Daniel A. Marinho1,2, Nuno D. Garrido ${ }^{2,3}$, Liliane S. Morgado ${ }^{4}$, Aldo M. \\ Costa ${ }^{1,2,5^{*}}$
}

\begin{abstract}
One of the key factors in the swimming teaching-learning process seems to be the variation of water's depth. However, there are almost no studies about this topic and the existing ones usually follow a basic approach and with no control of the educational program used. It was our purpose to determine the effect of deep versus shallow water differences on developing pre-schoolers' aquatic skills after 6 months of practice. Twenty-one Portuguese school-aged children of both genders ( $4.70 \pm 0.51 \mathrm{yrs}$.), inexperienced in aquatic programs, participated in this study. The children were divided into two groups performing a similar aquatic program but in a different water depth: shallow water $(n=10)$ and deep water $(n=11)$. Each participant was evaluated twice for their aquatic readiness using an observation check list of 17 aquatic motor skills: during the first session (T0) and after six months of practice (two sessions per week with a total of 48 sessions) (T1). The aquatic proficiency on each skill was compared between the groups and a stepwise discriminant analysis was conducted to predict the conditions with higher or lower aquatic competence. Results suggested that swimming practice contributed positively to improvements on several basic aquatic skills, in both groups. The results showed that shallow water group managed to acquire a higher degree of aquatic competence particularly in five basic aquatic skills $(\mathrm{p}<.05)$ : breath control combined with face immersion and eye opening; horizontal buoyancy; body position at ventral gliding; body position at dorsal gliding; leg kick with breath control at ventral body position, without any flutter device. The discriminant function revealed a significant association between both groups and four included factors (aquatic skills) $(\mathrm{p}<.001)$, accounting for $88 \%$ between group variability. The body position at ventral gliding was the main relevant predictor $(\mathrm{r}=0.535)$. Shallow water swimming lessons generated greater aquatic competence in preschool children after a period of 6 months of practice.

Keywords: Elite aquatic skills, teaching methods, children; shallow water, deep water
\end{abstract}

\section{INTRODUCTION}

Swimming is not considered a static personal ability (Langendorfer, 2014); instead, it implies an acquisition process, through practice and experience, which is built on a previous state of autonomy, confidence and satisfaction in the aquatic environment. Hence, aquatic competence is considered a bio-behavioural assumption of learning more complex and specialized aquatic skills, which also includes swimming strokes (Parker \& Blanksby, 1997; Warda, 2003). This conceptual understanding of aquatic competence is perhaps the most important in recent decades regarding swimming learning (Langerdorfer \&
Bruya, 1995; Moreno \& Sanmartín, 1998). It provided a coherent pedagogical foundation to reshape the "thinking processes of teachers" and therefore on what is tough and how is tough (Clark \& Peterson, 1986). However, there are still several pedagogical issues unanswered, mainly related with the process of swimming teaching and its results.

The uniqueness of the practice environment makes swimming a challenge for initiation of a constructive approach to teaching (Light \& Wallian, 2008). Although constructivism is not a prescription for teaching (Fosnot \& Perry, 1996), it is necessary to consider the teacher's role to

\footnotetext{
Manuscript received at August 25 $5^{\text {th }} 2017$; Accepted at December $10^{\text {th }} 2017$

${ }^{1}$ Department of Sports Sciences, University of Beira Interior, Covilhã, Portugal

${ }^{2}$ Research Centre in Sports, Health and Human Development, CIDESD, Portugal

${ }^{3}$ Department of Sports Sciences, Exercise and Health, University of Trás-os-Montes e Alto Douro, Vila Real, Portugal

${ }^{4}$ University of Liège, Liège, Belgium

${ }^{5}$ Health Science Research Center, CICS-UBI, Covilhã, Portugal

* Corresponding author: University of Beira Interior, Department of Sports Sciences, Rua Marquês d'Ávila e Bolama, 6201-001

Covilhã, Portugal E-mail: amcosta@ubi.pt
} 
provide optimal opportunities for learning. Therefore, proper environment conditions in a swimming pool can be particularly crucial to learning with effectiveness. One determinant factor seems to be the variation of the water depth (Costa et al., 2012). Indeed, aquatic readiness programs for young children can be performed in shallow water (usually from 0.65 to 1.00 meter deep), usually in the beginning of the process, or in deep water (usually from 1.00 meter to 2.00 meters deep), in the later stages. By decision of the swimming instructor or mere lack of structural alternative, there are aquatic programs for children (for utilitarian or formal educational purposes) almost exclusively conducted in deep water. One of the few studies on this subject compared the deep and shallow water effect on developing pre-schoolers' aquatic skills after six, twelve and eighteen months of practice (Costa et al., 2012). The results suggested that water depth might affect the acquisition of some basic aquatic skills, at least up to six months practice. However, that was a cross-sectional study, observational, which does not provide definitive information on the causeeffect of the conditions compared.

Therefore, the purpose of our study was to analyse the differences on developing preschoolers' aquatic skills between deep and shallow water aquatic programs after six months of practice. It is hypothesized that the shallow water program (while applying a controlled methodological approach) may induce an acquisition of basic aquatic skills at a higher level of proficiency.

\section{METHOD}

\section{Participants}

Participated in the present study 21 elementary school-aged children of both genders $(4.70 \pm 0.51 \mathrm{yrs}$.) with no previous experience in aquatic programs. The children were divided into two distinct classes with a similar aquatic program but performed on a different water depth environment: ten and 11 children performed all the swimming lessons in shallow water and deep water, respectively.

The swimming school board and the local Research Ethics Committee approved the experimental procedures, ensuring compliance with the declaration of Helsinki. The children's parents were informed about the study design and procedures and a written informed consent was signed. Data confidentiality was guaranteed, as well as their anonymity during the treatment process and analysis.

\section{Aquatic readiness assessment}

All children were evaluated twice for their aquatic readiness using an observation checklist of 17 aquatic motor skills based on Langerdorfer and Bruya (1995) and already applied by Costa et al. (2012): during the first session (T0) and after six months of practice (two sessions per week: 48 sessions; T1). The aquatic motor skills assessed were the following: water entry (Sk1); water orientation and adjustment at vertical position (Sk2); breath control - immersion of the face and eye opening (Sk3); horizontal buoyancy (Sk4); body position at ventral gliding (Sk5); body position at dorsal gliding (Sk6); body position at longitudinal rotation in gliding (Sk7); body position at front and back somersaults (Sk8); leg kick with breath control at ventral body position, with flutter boards (Sk9); and without any flutter device, (Sk10); leg kick with breath control at dorsal body position with flutter boards (Sk11); and without any flutter device (Sk12); feet-first entry (Sk13); head-first entry (Sk14); Autonomous in deep water (legs and arms displacement) (Sk15); vertical buoyancy at deep water (Sk16); deep water immersion (Sk17). Each one of these skills was divided into increasing levels of complexity (three, four or five levels, depending on the categorical skill) as suggested by Langendorfer and Bruya (1995): enable to perform at stage one, rudimentary movements at stage two (or three) and fundamental movements at stage three (or even four or five) that precede the specific motor skill acquisition. The children had three attempts to achieve the proposed exercises, as conducted by Costa et al. (2012).

\section{Swimming practice}

At the beginning of the study, all the children were in a state of total inaptness to the aquatic environment. The swimming sessions took place 
at the same time of the day, twice a week, with 45 min duration (between $6 \mathrm{~h} 45$ and $7 \mathrm{~h} 30$ p.m.). The shallow water sessions were carried out in a 0.70 $\mathrm{cm}$ water depth, with the water temperature at $31^{\circ} \mathrm{C}$, the air temperature at $29^{\circ} \mathrm{C}$ and a relative humidity of $65 \%$. The deep water sessions occurred in a 1.30 meter water depth, with a water temperature of temperature $29^{\circ} \mathrm{C}$, air temperature of $29^{\circ} \mathrm{C}$ and the relative humidity was $65 \%$.

Both aquatic programs aimed to improve children's aquatic readiness by teaching basic aquatic skills. The number of students in each class was reduced to increase the useful time of the lesson and minimize practice waiting time among students. The swimming teacher was the same in both groups. Therefore, the teaching methods and the skills developed in each class were similar and based on the literature guidelines (e.g., Canossa, Fernandes, Carmo, Andrade, \& Soares, 2007; Langendorfer \& Bruya, 1995;). Table 1 shows how the aquatic skills were sequenced over the six months of teaching.

Table 1

Aquatic program characteristics conducted in both water deep environment.

\begin{tabular}{|c|c|c|c|c|c|c|c|c|c|c|c|c|c|c|c|c|c|c|c|c|c|c|c|c|}
\hline Month & \multicolumn{4}{|c|}{1} & \multicolumn{4}{|c|}{2} & \multicolumn{4}{|c|}{3} & \multicolumn{4}{|c|}{4} & \multicolumn{4}{|c|}{5} & \multicolumn{4}{|c|}{6} \\
\hline Week & 1 & 2 & 3 & 4 & 5 & 6 & 7 & 8 & 9 & 10 & 11 & 12 & 13 & 14 & 15 & 16 & 17 & 18 & 19 & 20 & 1 & 22 & 23 & 24 \\
\hline Sk1 & $\uparrow$ & $\uparrow$ & $\uparrow$ & $\uparrow$ & $\pi$ & $\pi$ & $\pi$ & $\pi$ & $\rightarrow$ & $\leftrightarrow$ & $\leftrightarrow$ & $\leftrightarrow$ & $\rightarrow$ & $\leftrightarrow$ & $\leftrightarrow$ & $\rightarrow$ & $\rightarrow$ & $\leftrightarrow$ & $\leftrightarrow$ & $\leftarrow$ & $\rightarrow$ & $\leftrightarrow$ & $\leftrightarrow$ & $\leftrightarrow$ \\
\hline Sk2 & $\uparrow$ & $\uparrow$ & $\uparrow$ & $\uparrow$ & $\lambda$ & $\lambda$ & $\lambda$ & $\pi$ & & $\leftrightarrow$ & $\leftrightarrow$ & $\leftarrow$ & $\rightarrow$ & $\leftrightarrow$ & $\leftrightarrow$ & $\leftrightarrow$ & $\rightarrow$ & $\leftrightarrow$ & $\leftrightarrow$ & $\leftrightarrow$ & $\rightarrow$ & $\leftrightarrow$ & $\leftrightarrow$ & $\leftrightarrow$ \\
\hline $\mathrm{Sk3}$ & $\uparrow$ & $\uparrow$ & $\uparrow$ & $\uparrow$ & $\uparrow$ & $\uparrow$ & $\uparrow$ & $\uparrow$ & $\nearrow$ & 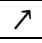 & $\lambda$ & $\pi$ & $\lambda$ & $\nearrow$ & $\pi$ & $\lambda$ & 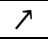 & $\pi$ & $\pi$ & $\lambda$ & $\pi$ & $\lambda$ & $\lambda$ & $\pi$ \\
\hline Sk4 & $\uparrow$ & $\uparrow$ & $\uparrow$ & $\uparrow$ & $\uparrow$ & $\uparrow$ & $\uparrow$ & $\uparrow$ & $\uparrow$ & 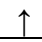 & $\uparrow$ & $\uparrow$ & $\nearrow$ & $\nearrow$ & $\lambda$ & $\pi$ & $\leftrightarrow$ & $\leftrightarrow$ & $\leftrightarrow$ & $\leftrightarrow$ & $\leftrightarrow$ & $\leftrightarrow$ & $\leftrightarrow$ & $\leftrightarrow$ \\
\hline Sk5 & $\uparrow$ & $\uparrow$ & $\uparrow$ & $\uparrow$ & $\uparrow$ & $\uparrow$ & $\uparrow$ & $\uparrow$ & $\uparrow$ & $\uparrow$ & $\uparrow$ & $\uparrow$ & $\uparrow$ & 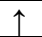 & $\uparrow$ & $\uparrow$ & $\uparrow$ & $\uparrow$ & $\uparrow$ & $\uparrow$ & 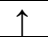 & $\uparrow$ & $\uparrow$ & $\uparrow$ \\
\hline Sk6 & $\uparrow$ & $\uparrow$ & $\uparrow$ & $\uparrow$ & $\uparrow$ & $\uparrow$ & $\uparrow$ & $\uparrow$ & & $\uparrow$ & $\uparrow$ & & & & 1 & 1 & 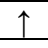 & 1 & 1 & 工 & & I & $\uparrow$ & $\uparrow$ \\
\hline Sk7 & $\square$ & $\square$ & $\square$ & $\square$ & $\uparrow$ & $\uparrow$ & $\uparrow$ & $\uparrow$ & & $\uparrow$ & 1 & $\uparrow$ & 1 & & $\uparrow$ & 1 & $\pi$ & $\lambda$ & $\lambda$ & $\pi$ & $\pi$ & $\lambda$ & $\pi$ & $\pi$ \\
\hline Sk8 & $\square$ & $\square$ & $\square$ & $\square$ & $\square$ & $\square$ & $\square$ & $\square$ & I & $\uparrow$ & $\uparrow$ & $\uparrow$ & $\uparrow$ & & $\uparrow$ & $\uparrow$ & $\uparrow$ & $\uparrow$ & $\uparrow$ & $\uparrow$ & $\lambda$ & $\lambda$ & $\lambda$ & $\lambda$ \\
\hline Sk9 & $\square$ & $\square$ & $\square$ & $\square$ & $\square$ & $\square$ & $\square$ & $\square$ & $\square$ & $\square$ & $\uparrow$ & $\uparrow$ & $\uparrow$ & & $\uparrow$ & $\uparrow$ & $\uparrow$ & $T$ & $\uparrow$ & $\uparrow$ & $\pi$ & $\lambda$ & $\pi$ & $\pi$ \\
\hline Sk10 & $\square$ & $\square$ & $\uparrow$ & $\uparrow$ & $\uparrow$ & $\uparrow$ & $\uparrow$ & $\uparrow$ & 1 & $\uparrow$ & $\uparrow$ & $\uparrow$ & $\uparrow$ & & $\uparrow$ & $\pi$ & $\lambda$ & $r$ & $\pi$ & $\pi$ & $\rightarrow$ & $\leftrightarrow$ & $\leftrightarrow$ & $\leftrightarrow$ \\
\hline Sk11 & $\square$ & $\square$ & $\square$ & $\square$ & $\square$ & $\square$ & $\square$ & $\square$ & $\square$ & $\square$ & $\uparrow$ & $\uparrow$ & $\uparrow$ & $\uparrow$ & $\uparrow$ & $\uparrow$ & $\uparrow$ & $\uparrow$ & $\uparrow$ & $\uparrow$ & $\pi$ & $\lambda$ & $\pi$ & $\pi$ \\
\hline Sk12 & $\square$ & $\square$ & $\uparrow$ & $\uparrow$ & $\uparrow$ & $\uparrow$ & $\uparrow$ & $\uparrow$ & $\uparrow$ & $\uparrow$ & $\uparrow$ & $\uparrow$ & 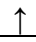 & & $\uparrow$ & $\lambda$ & $\lambda$ & $\lambda$ & $\lambda$ & $\lambda$ & $\rightarrow$ & $\leftrightarrow$ & $\leftrightarrow$ & $\leftrightarrow$ \\
\hline Sk13 & $\uparrow$ & $\uparrow$ & $\uparrow$ & $\uparrow$ & $\uparrow$ & $\uparrow$ & $\uparrow$ & $\uparrow$ & $\uparrow$ & $\pi$ & $\pi$ & 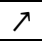 & 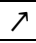 & 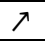 & 7 & $\pi$ & $\lambda$ & $\leftrightarrow$ & $\leftrightarrow$ & $\leftrightarrow$ & $\rightarrow$ & $\leftrightarrow$ & $\leftrightarrow$ & $\leftrightarrow$ \\
\hline Sk14 & $\square$ & $\square$ & $\square$ & $\square$ & $\uparrow$ & $\uparrow$ & $\uparrow$ & $\uparrow$ & $\uparrow$ & $\uparrow$ & $\uparrow$ & $\uparrow$ & 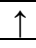 & $\uparrow$ & 7 & $\pi$ & $\pi$ & $\pi$ & $\lambda$ & $\lambda$ & $\leftrightarrow$ & $\leftrightarrow$ & $\leftrightarrow$ & $\leftrightarrow$ \\
\hline Sk15* & $\square$ & $\square$ & $\uparrow$ & $\uparrow$ & $\uparrow$ & $\uparrow$ & $\uparrow$ & $\uparrow$ & & 1 & $\uparrow$ & & $\uparrow$ & & 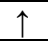 & $\pi$ & $\pi$ & 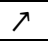 & $\pi$ & $\lambda$ & $\leftrightarrow$ & $\leftrightarrow$ & $\leftrightarrow$ & $\leftrightarrow$ \\
\hline Sk16* & $\uparrow$ & $\uparrow$ & $\uparrow$ & $\uparrow$ & $\uparrow$ & $\uparrow$ & $\uparrow$ & 1 & 1 & 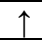 & $\uparrow$ & $\uparrow$ & 7 & $\pi$ & $\lambda$ & $\pi$ & $\leftrightarrow$ & $\leftrightarrow$ & $\leftrightarrow$ & $\leftrightarrow$ & $\leftrightarrow$ & $\leftrightarrow$ & $\leftrightarrow$ & $\leftrightarrow$ \\
\hline Sk17* & $\square$ & $\square$ & $\square$ & $\square$ & $\square$ & $\square$ & $\square$ & $\square$ & $\uparrow$ & $\uparrow$ & $\uparrow$ & $\uparrow$ & $\uparrow$ & $\uparrow$ & $\uparrow$ & $\uparrow$ & $\uparrow$ & $\uparrow$ & $\uparrow$ & $\uparrow$ & $\lambda$ & $\pi$ & $\pi$ & \\
\hline
\end{tabular}

Legend: ๑, Aquatic skill not developed; $\uparrow$, Aquatic skill highly developed; $\nearrow$, Aquatic skill moderately developed; $\Theta$, Aquatic skill not directly development but consider pre-requisite.

Teaching style shifted from absolute control (command and task style) to more indirect teaching style, best known as guided discovery (Mosston \& Ashworth, 1990). Indeed, the students mostly performed analytical tasks to develop basic aquatics skills in both aquatic environments. However, ludic tasks were also included, leading the child to discover a predetermined "aquatic motor target" in response to a sequence of "problems" presented by the teacher. Sometimes it was necessary to adjust certain aquatic tasks due to physical embarrassment imposed by depth. As such, we had to make minor changes to the task organization (i.e., smaller groups and slight changes to certain rules of play) and use some floating didactic material. The following didactic and floating material was used: didactic-puzzles, towers, slides, mattresses, overflow arches, rings, floating-arches, balls, small boards and noodles.

\section{Statistical analysis}

Standard statistical methods were used for the calculation of means and standard deviations. The $t$ test was used to compare the differences in aquatic proficiency (on each skill) between groups. The effect size was calculated using Cohen's d (Cohen, 1988). A stepwise discriminant analysis was also conducted with $\Lambda$ wilk's method to build a predictive model for group membership (aquatic competence for shallow and deep water students). Predictor variables were the 17 aquatic motor skills previously described. Box's M variance-covariance 
matrices were used to test the multivariate homogeneity. The level of statistical significance was set at $\mathrm{p} \leq .05$.

\section{RESULTS}

Table 2 presents the aquatic skills acquired by shallow water and deep water students during six months of practice.

Table 2

Aquatic skills acquired (mean $\pm S D$ ) by shallow-water and deep-water students after six months of practice.

\begin{tabular}{cc|cccc|cccc}
\hline \multirow{2}{*}{ Skill } & $\begin{array}{c}\text { Levels of } \\
\text { complexity }\end{array}$ & $\begin{array}{c}\text { Shallow } \\
\text { Water } \\
\text { (n=11) }\end{array}$ & $\begin{array}{c}\text { Deep Water } \\
\text { (n=10) }\end{array}$ & P-value & $\begin{array}{c}\text { Cohen's } \\
\text { d }\end{array}$ & $\begin{array}{c}\text { Shallow } \\
\text { Water } \\
\text { (n=11) }\end{array}$ & $\begin{array}{c}\text { Deep Water } \\
\text { (n=10) }\end{array}$ & $\begin{array}{c}\text { P-value } \\
\text { Cohen's } \\
\text { d }\end{array}$ \\
\hline$S k 1$ & 1 to 3 & $1.091 \pm .302$ & $1.100 \pm .316$ & .947 & .029 & $3.000 \pm .000$ & $2.900 \pm .316$ & .306 & .448 \\
\hline$S k 2$ & 1 to 3 & $1.273 \pm .467$ & $1.000 \pm .000$ & .081 & .827 & $3.000 \pm .000$ & $0.000 \pm .000$ & - & - \\
\hline$S k 3$ & 1 to 5 & $1.000 \pm .000$ & $1.000 \pm 000$ & - & - & $4.189 \pm .879$ & $3.100 \pm .137$ & $.042^{*}$ & 1.73 \\
\hline$S k 4$ & 1 to 4 & $1.000 \pm .000$ & $1.000 \pm .000$ & - & - & $2.636 \pm .120$ & $1.500 \pm .850$ & $.018^{*}$ & 1.87 \\
\hline$S k 5$ & 1 to 4 & $1.000 \pm .000$ & $1.000 \pm .000$ & - & - & $2.727 \pm .647$ & $1.200 \pm .422$ & $.000^{*}$ & 2.79 \\
\hline$S k 6$ & 1 to 4 & $1.000 \pm .000$ & $1.000 \pm .000$ & - & - & $2.090 \pm .831$ & $1.100 \pm .316$ & $.002^{*}$ & 1.57 \\
\hline$S k 7$ & 1 to 3 & $1.000 \pm .000$ & $1.000 \pm .000$ & - & - & $1.455 \pm .522$ & $1.300 \pm .483$ & .491 & .308 \\
\hline$S k 8$ & 1 to 4 & $1.000 \pm .000$ & $1.000 \pm .000$ & - & - & $1.000 \pm .000$ & $1.000 \pm .000$ & - & - \\
\hline$S k 9$ & 1 to 4 & $1.000 \pm .000$ & $1.000 \pm .000$ & - & - & $2.455 \pm 522$ & $2.100 \pm .316$ & .079 & .823 \\
\hline$S k 10$ & 1 to 4 & $1.000 \pm .000$ & $1.000+.000$ & - & - & $2.000 \pm .632$ & $1.400 \pm .516$ & $.029^{*}$ & 1.04 \\
\hline$S k 11$ & 1 to 4 & $1.000 \pm .000$ & $1.000 \pm .000$ & - & - & $2.091 \pm .701$ & $1.700 \pm .675$ & .209 & .568 \\
\hline$S k 12$ & 1 to 4 & $1.000 \pm .000$ & $1.000 \pm .000$ & - & - & $1.818 \pm .874$ & $1.200 \pm .422$ & .057 & .900 \\
\hline$S k 13$ & 1 to 3 & $1.000 \pm .000$ & $1.000 \pm .000$ & - & - & $2.364 \pm .505$ & $1.800 \pm .789$ & .064 & .851 \\
\hline$S k 14$ & 1 to 3 & $1.000 \pm .000$ & $1.000 \pm .000$ & - & - & $1.727 \pm .467$ & $1.300 \pm .675$ & .105 & .736 \\
\hline$S k 15$ & 1 to 3 & $1.000 \pm .000$ & $1.000 \pm .000$ & - & - & $1.364 \pm .505$ & $1.500 \pm .527$ & .552 & .264 \\
\hline$S k 16$ & 1 to 5 & $1.000 \pm .000$ & $1.000 \pm .000$ & - & - & $1.364 \pm .505$ & $1.700 \pm .483$ & .136 & .680 \\
\hline$S k 17$ & 1 to 4 & $1.000 \pm .000$ & $1.000 \pm .000$ & - & - & $1.182 \pm .405$ & $1.300 \pm .483$ & .549 & .265 \\
\hline & & & & & & & & & \\
\hline
\end{tabular}

At the beginning of this study (T0), no differences were found in aquatic readiness between shallow and deep water. The students were not adapted to the aquatic environment and their aquatic motor proficiency was zero in all aquatic skills. After six months of practice there were differences between the means of both groups in five aquatic skills: Sk3, Sk4, Sk5 Sk6 and Sk10.

The stepwise discriminant analysis was used to determine which aquatic skills discriminate between both groups after six months of practice. The step-by-step model of discrimination was built with four steps, including the following aquatic skills: Sk5 $(\mathrm{F}=40.151, \mathrm{p}<.001)$; Sk16 $(\mathrm{F}=34.254, \mathrm{p} .001) ; \mathrm{Sk} 15(\mathrm{~F}=29.237, \mathrm{p}<.001)$ and Sk13 $(\mathrm{F}=29.489, \mathrm{p}<.001)$. The canonical discriminant function analysis revealed a significant association between both groups and all included factors, accounting for $(0.938)^{\wedge} 2=88 \%$ between group variability $(\Lambda=0.119$, Qui^ $2=36.124, \mathrm{p}<.001)$.

Table 3 shows the pooled within-groups correlations between discriminating variables and standardized canonical discriminant functions. The variables are ordered by absolute size of correlation within function; Sk5 is the main predictor with a relevant absolute size of correlation within function $(r=0.535)$. The functions at groups' centroids shows that shallow-water students have a mean of 2.46 $( \pm 1.064)$ while deep-water students produce a mean of $-2.71( \pm 0.924) ; 100 \%$ of students were correctly classified.

\section{Table 3}

Structure matrix for shallow-water and deep-water students after six months of practice.

\begin{tabular}{c|c}
\hline Skill & Function \\
\hline S5 & .535 \\
\hline S9 & .296 \\
\hline S6 $^{\mathrm{a}}$ & .284 \\
\hline $\mathrm{S}^{\mathrm{a}}$ & .261 \\
\hline $\mathrm{S} 12^{\mathrm{a}}$ & .227 \\
\hline $\mathrm{S} 10^{\mathrm{a}}$ & -.199 \\
\hline $\mathrm{S} 13$ & .166 \\
\hline $\mathrm{S} 1^{\mathrm{a}}$ & -.159 \\
\hline $\mathrm{S} 7^{\mathrm{a}}$ & .158 \\
\hline $\mathrm{S} 14$ & .144 \\
\hline $\mathrm{S} 16$ & -.132 \\
\hline $\mathrm{S} 15^{\mathrm{a}}$ & .124 \\
\hline $\mathrm{S} 4^{\mathrm{a}}$ & .048 \\
\hline $\mathrm{S} 17^{\mathrm{a}}$ & -.032 \\
\hline $\mathrm{S} 11^{\mathrm{a}}$ & -.029
\end{tabular}

Legend: a - Variable not included in the step-by-step model. 


\section{DISCUSSION}

The first aim of the present study was to analyse the development of basic aquatic skills and to compare the effect of swimming practices in two distinct swimming pool environments (deep and shallow swimming pools). Results showed positive effects of swimming practice in children's aquatic competence from both sessions' types. However, shallow water students managed to acquire greater aquatic competence in nearly all aquatic skills measured after six months of practice.

The swimming programs were more than just the simple acquisition of new motor patterns that allow moving inside the aquatic environment (Langendorfer \& Bruya, 1995; Martins et al., 2010). These are based on the need to adjust the motor behaviour of the child in the water, helping to understand the particularities of the aquatic environment, specifically the lower gravity and viscosity (Holmér, 1974). Therefore, the enjoyment for swimming practice is associated with the notion of trust about their own security in the new environment (Brenner, Saluja, \& Smith, 2003; Velasco, 1994).

In the initial phase, the confidence of the child in the aquatic environment could be easily affected when, for instance, the water depth of exercitation is changed. This constrain caused by the depth of the pool could influence their autonomy. The current study did not have the purpose to study the variability of the pedagogic intervention or of the student's motor behaviour in both pool environments (for that see Costa et al., 2012). Nevertheless, it is our perception that the water depth seems to be an inhibitory factor to discover the aquatic environment and its particularities. Thus, the water depth could constrain the students' creativity in the resolution of major motor problems caused by the aquatic environment, at least in the early stages of familiarization. Although our effort to provide identical pedagogic experiences in both environments, for safety reasons it is understandable that teaching in deep water could be less student-centred. There is a need to adapt the swimming tasks due to the mandatory use of float materials and the lack of confidence of the student. Therefore, the teaching methods in this condition tended to be more traditional (Mosston, 1992). This occurs at least in the initial phase of development, in which the students' actions are always derived from the teacher decisions. Considering a complete understanding of the concept of aquatic competence, it is not imperative that there is only one response to similar situations (Moreno \& Sanmartín, 1998). Thus, we believe that different water depths during swimming lessons inevitably provide different psychomotor experiences. Our results, as we discuss below, seem to support such reasoning.

As reported in table 12, those children who attended to shallow water lessons presented greater level of aquatic competence in several skills, namely: breath control - immersion of the face and eye opening (Sk3), horizontal buoyancy (Sk4), body position at ventral gliding (Sk5), body position at dorsal gliding (SK6), and leg kick with breath control at ventral body position, without any flutter device (Sk10). These results are consistent with the data reported by Costa et al. (2012); although these authors reported differences between both session types after six months of practices also in the following skills: water entry (Sk1); body position at longitudinal rotation in gliding (Sk7); body position at front and back somersaults (Sk8); leg kick with breath control at ventral body position, with flutter boards (Sk9); leg kick with breath control at dorsal body position with flutter boards (Sk11); and without any flutter device (Sk12); feet-first entry (Sk13); head-first entry (Sk14); vertical buoyancy at deep water (Sk16); deep water immersion (Sk17). These substantial differences in the acquired aquatic competence as reported by Costa et al. (2012) can derive from the variability of the teaching intervention, given that teachers were not the same in both sessions' types.

The discriminant analysis showed that the Sk5 was the main predictor with significant correlation within function, consistent with the data reported by Costa et al. (2012). This could be related with the lower opportunity to develop the glide in ventral/dorsal position and in different depths in the early learning stages in deep water condition. Probably, the use of 
floating devices caused some changes in the horizontal position and an unreal sense of buoyancy (Blanksby, Parker, Bradley, \& Ong, 1995).

Our results showed that six months of practices in both conditions allowed students to develop the aquatic readiness of the majority of the aquatic basic skills, with exception to body position at front and back somersaults (SK8), which is in accordance with the results presented by Costa et al. (2012). Moreover, the body position at longitudinal rotation in gliding ( $\mathrm{Sk} 7$ ) was nearly learned in both conditions probably because it is an aquatic motor skill conditioned by the previous acquisition of other basic skills, as the water entry, glide, respiration and static vertical balance in deep water. It would be also important to refer that six months of practice in both swimming pool environments were not sufficient to achieve mastery on all aquatic skills. Similar data have been reported by Costa et al. (2012) after six and 12 months of aquatic practice.

Despite the importance of the results presented to the scientific and technical community, some limitations should be addressed to the current study. Firstly, it was only possible to access the aquatic competence of the students after six months of practice, ensuring the inclusion criterion of keeping the same teacher. Also, the limitation regarding the number of participants observed, conditioning the conclusions and the extrapolation of the results to other subjects. The effect size was used to better control and analyse the differences obtained. Thirdly, no data about the activity time devoted to swimming practice in both session types. Although the number of students has been reduced to enable high activity time in both sessions, we recognize that differences regarding this variable may exist. Future studies should assess the variation of activity time regarding to different learning contexts, number of students and teaching styles. Future studies should also seek to analyse the effectiveness of concurrent water depth environments programs (shallow and deep water) and the question of timing and dosing of swimming practice.

\section{CONCLUSIONS}

In conclusion, the present study suggests that a shallow water environment is more suitable for the development of basic aquatic skills in preschool children. The stepwise discriminant analysis revealed a significant association between both session types and four included aquatic skills for six months of practice; the body position at ventral gliding seems to be the main significant predictor. This could mean that aquatic skills at the children beginner's level should be learnt in a shallow water swimming pool and deep water programs should be carefully planned to stimulate certain skills (i.e. body gliding) that seems to be differently exercised in both pool environments.

\footnotetext{
Acknowledgments:

To all the parents and children, for their kind participation in this research.

To NanoSTIMA: Macro-to-Nano Human Sensing: Towards Integrated Multimodal Health Monitoring and Analytics(NORTE-01-0145-FEDER-000016), cofinanced by the Fundo Europeu de Desenvolvimento Regional (FEDER) through NORTE 2020
}

Conflict of interests:

Nothing to declare.

\section{Funding: \\ Nothing to declare.}

\section{REFERENCES}

Blanksby, B. A., Parker, H. E., Bradley, S., \& Ong, V. (1995). Children's readiness for learning front crawl swimming. The Australian Journal of Science and Medicine in Sport, 27(2), 34-37.

Brenner, R. A., Saluja, G., \& Smith, G. S. (2003). Swimming lessons, swimming ability, and the risk of drowning. Injury Control and Safety Promotion, 10(4), 211-215.

Canossa, S., Fernandes, R. J., Carmo, C., Andrade, A., \& Soares, S. M. (2007). Ensino multidisciplinar em natação: reflexão metodológica e proposta de lista de verificação". Motricidade, 3(4), 82-99. doi: 10.6063/motricidade.3(4).656

Clark, C., \& Peterson, P. (1986). Teacher's thought processes. In M. Wittrock (Ed), Handbook of Research on Teaching (pp. 255-296). New York, NY: Macmillan Publishing Company.

Cohen, J. (1988). Statistical power analysis for the behavioral sciences. Hillsdale, NJ: Lawrence Earlbaum Associates. 
Costa, A. M., Marinho, D. A., Rocha, H., Silva, A. J., Barbosa, T. M., Ferreira, S. S., \& Martins, M. (2012). Deep and Shallow Water Effects on Developing Preschoolers' Aquatic Skills. Journal of Human Kinetics, 32, 211-219. doi: 10.2478/v10078-012-0037-1.

Fosnot, C. T., \& Perry, R. S. (1996). Constructivism: A psychological theory of learning. In C. T. Fosnot (Ed.), Constructivism: Theory, Perspectives, and Practice (pp. 8-33). New York: Teachers College Press.

Holmér, I. (1974). Physiology of swimming man. Acta Physiologica Scandinavica, Suplementum 407, 1-55.

Langendorfer, S. (2014). Swimming Learning Standards: An International Perspective. $34^{\circ}$ Congreso de la Asociación Española de Técnicos de Natación. Granada, Spain.

Langerdorfer, S., \& Bruya, L. (1995). Aquatic Readiness: Developing Water Competence in Young Children. Champaign, IL: Human Kinetics.

Light, R., \& Wallian, N. (2008). A ConstructivistInformed Approach to Teaching Swimming. Quest, 60(3): 387-404. doi: $10.1080 / 00336297.2008 .10483588$
Martins, M., Silva, A. J., Marinho, D. A., Pereira, A., Moreira, A. M., Sarmento, P., \& Barbosa, T. M. (2010). Assessment of heart rate during infants swim session. International SportsMed Journal, $11(3), 336-344$.

Moreno, J., \& M. Sanmartín (1998). Bases metodológicas para el aprendizaje de las actividades acuáticas educativas. Barcelona, Spain: INDE Publicaciones.

Mosston, M. (1992). Tug-O-War, no more: Meeting teaching-learning objectives using the spectrum of teaching styles. Journal of Physical Education, Recreation \& Dance, 63(1), 27-56.

Mosston, M., \& S. Ashworth (1990). The Spectrum of Teaching Styles. From Command to Discovery. White Plains, NY: Longman.

Parker, H. E., \& B. A. Blanksby (1997). Starting age and aquatic skill learning in young children: mastery of prerequisite water confidence and basic aquatic locomotion skills. Australian Journal of Science and Medicine in Sport, 29(3), 83-87.

Velasco, C.G. (1994). Natação segundo a psicomotricidade. Rio de Janeiro, Brasil: Editora Sprint.

Warda, L. (2003). Swimming lessons for infants and toddlers. Pediatrics \& Child Health, 8(2), 113-114.

All content of Journal Motricidade is licensed under Creative Commons, except when otherwise specified and in content retrieved from other bibliographic sources. 